\title{
Performance Analysis of Multicarrier CDMA Systems With Frequency Offsets and Random Spreading Under Optimum Combining
}

\author{
Feng-Tsun Chien, Chien-Hwa Hwang, and C.-C. Jay Kuo, Fellow, IEEE
}

\begin{abstract}
The carrier-frequency offset effect on the performance of asynchronous multicarrier direct-sequence code-division multiple-access (MC-DS-CDMA) systems with aperiodic random spreading and correlated Rayleigh fading is studied in this paper. We obtain the optimum combining filter that maximizes the signal-to-interference-plus-noise ratio (SINR) of the combined statistics and exploits correlated information among subchannels. A closed-form expression for the unconditional covariance matrix of the interference-plus-noise vector, which forms the basis of our theoretical analysis of the maximum SINR and the average bit error probability formula, is derived by averaging several random parameters including asynchronous delays, correlated Rayleigh fading, and signature sequences. The analytic results obtained are applicable to MC-CDMA with appropriate modifications. Furthermore, we show that the MC-CDMA system with a common random signature sequence over all subcarriers for a given user outperforms that with distinct sequences over different subcarriers. Finally, the performance of MC-CDMA systems using the optimum combining technique is compared with that of different combining filters in the simulation.
\end{abstract}

Index Terms-Asynchronous transmission, carrier-frequency offset (CFO), code-division multiple access (CDMA), multicarrier CDMA (MC-CDMA), random signature sequence.

\section{INTRODUCTION}

$\mathbf{N}$ EXT-GENERATION wireless systems featuring interactive multimedia communications require high-rate-data transmissions in diverse mobile environments. The underlying wideband nature makes the overall system prone to a hostile frequency-selective multipath channel condition. To enhance detection performance, the design of a robust system that is capable of mitigating channel frequency selectivity as well as exploiting the inherent multipath diversity is essential.

Paper approved by M. Chiani, the Editor for Transmission Systems for the IEEE Communications Society. Manuscript received October 9, 2004; revised June 15, 2005. This work was supported by the Integrated Media Systems Center, a National Science Foundation Engineering Research Center, under Cooperative Agreement EEC-9529152. This work was presented in part at the IEEE Global Telecommunications Conference, San Francisco, CA, December 2003.

F.-T. Chien is with the Department of Electronics Engineering, National Chiao Tung University, Hsunchu 30050, Taiwan, R.O.C. (e-mail: ftchien@ mail.nctu.edu.tw).

C.-H. Hwang is with the Institute of Communications Engineering, National Tsing Hua University, Hsinchu 30013, Taiwan, R.O.C. (e-mail: chhwang@ee. nthu.edu.tw).

C.-C. Jay Kuo is with the Integrated Media Systems Center and the Department of Electrical Engineering, University of Southern California, Los Angeles, CA 90089-2564 USA (e-mail: cckuo@ sipi.usc.edu).

Digital Object Identifier 10.1109/TCOMM.2006.873094
However, this often yields a complicated receiver structure in a single-carrier communication system. In contrast, the multicarrier code-division multiple-access (MC-CDMA) modulation technique provides an effective and promising solution to this problem and serves as a strong candidate for future broadband wireless communications systems.

There are two types of MC-CDMA systems in the literature. One is the multicarrier-CDMA proposed in [1] to combat the severe indoor multipath effect. With a different spreading mechanism, Kondo and Milstein [2] proposed another system, called the MC-direct-sequence CDMA (MC-DS-CDMA) system, which is effective in suppressing partial-band interference. Both systems exhibit the capability of exploiting frequency diversity and mitigating the frequency-selective fading effect without the use of RAKE-structured receivers. To avoid confusion, we will use acronyms MC-CDMA and MC-DS-CDMA to refer to the corresponding systems for the rest of this paper, while multicarrier CDMA will be used when the description is applicable to both.

Although multicarrier CDMA systems have been justified by having appealing properties due to multicarrier modulation and CDMA techniques [3], they inherit some unpleasant drawbacks as well. For instance, the sensitivity to synchronization errors poses a great challenge on the system design. In particular, the carrier frequency offset (CFO), resulting from the Doppler shift or the oscillators frequency mismatch at the transmitter/receiver, destroys the orthogonality between any subcarrier pairs. This introduces off-band multiple-access interference (MAI) or, equivalently, intercarrier interference (ICI), which deteriorates the system performance significantly.

The CFO effect on the performance of multicarrier CDMA systems was studied by researchers before, e.g., [4]-[7]. In particular, the CFO effect on MC-CDMA and MC-DS-CDMA systems was analyzed using zero-forcing equalization under the additive white Gaussian noise (AWGN) channel in [4] and the Rayleigh fading channel in [5], where the fading among subchannels was assumed to be independent. The CFO effect on synchronous and asynchronous MC-CDMA systems with correlated subchannel fading was, respectively, investigated in [6] and [7], where the diversity combining coefficients were chosen to be the corresponding channel coefficients under the maximal ratio combining (MRC) criterion. All of the abovementioned work assumed periodic signature sequences, i.e., the same signature sequence is used for every symbol for a given user.

Since the use of aperiodic (random) spreading in CDMA systems can lower the probability of intentional intercepts 
and avoid unpleasant occasions of high correlations between signature sequences due to asynchronous delays, it is widely used in practical CDMA systems. It is interesting and valuable to investigate the $\mathrm{CFO}$ effect with random signature sequences. In this study, we conduct performance analysis for the uplink asynchronous MC-DS-CDMA system proposed in [2] with $\mathrm{CFO}$ and random spreading under optimum combining. The result obtained is also applicable to MC-CDMA with slight modifications by setting the time-domain spreading gain equal to one. In particular, we investigate the influence of two different signature sequences, i.e., identical spreading and distinct spreading over different subcarriers for a given user, which may correspond to the spreading mechanism employed by MC-DS-CDMA [2] and MC-CDMA [8] systems, respectively.

In this paper, we formulate the signal model of a multicarrier CDMA system in a vector-matrix form by incorporating several realistic factors such as asynchronous transmission, CFO, correlated fading among subchannels, and random spreading. A closed-form expression of the covariance matrix of the interference-plus-noise vector is derived by averaging all random parameters in the received signal, namely, asynchronous delays, CFOs, fading coefficients, and random signature sequences. Moreover, the bit error probability (BEP) formula is obtained based on the Gaussian assumption of the statistics contributed by the MAI and using the local-mean approximation approach presented in [8] and [9, App. E]. Finally, the performance of the proposed optimum receiver is compared with that of other combining filters proposed in the literature via computer simulation.

The remainder of this paper is organized as follows. The signal models at the transmitter, the channel, and the receiver of an asynchronous multicarrier CDMA system are presented in Section II. The closed-form solution for the combining filter maximizing the instantaneous SINR is derived in Section III. The BEP formula for the target system is given in Section IV. Simulation results, which verify the accuracy of the analysis and compare performance of several systems, are provided in Section V. Finally, concluding remarks are made in Section VI.

\section{SYSTEM MODEL}

\section{A. Transmitted Signal Model}

Consider the uplink of a multicarrier long-code CDMA system with bandwidth $W$ and $M$ subcarriers. For both MCCDMA and MC-DS-CDMA systems, the transmitted signal of user $k$ can be expressed in the complex analytic form as [10]

$$
s_{k}(t)=\sum_{m=1}^{M} \sqrt{\frac{2 P_{k}}{M}}\left(\sum_{i=-\infty}^{\infty} b_{k}(i) c_{k, m}\left(t-i T_{b}\right)\right) e^{j 2 \pi f_{m} t}
$$

where $b_{k}(i)$ denotes the $i$ th data symbol with duration $T_{b}, P_{k}$ is the average transmitted power, $c_{k, m}(t)$ is the normalized spreading waveform at the $m$ th subcarrier of user $k$, respectively, and $f_{m}=f_{0}+m(W / M)$ is the modulation frequency at the $m$ th subcarrier where $f_{0}$ is the reference frequency.

In an MC-DS-CDMA system, a set of $M$ distinct signature sequences are assigned to all users during every symbol interval.
As opposed to the spreading mechanism in [2], we allow the signature sequences over distinct subcarriers to be identical or to be different for each user [11], [12]. In particular, we are interested in two extreme cases where, for a given user, all of the $M$ distinct sequences are simultaneously employed or only one sequence is employed over all $M$ subcarriers at every symbol interval. The latter corresponds to the system proposed in [2]. Specifically, the spreading waveform $c_{k, m}\left(t-i T_{b}\right)$ in (1) is expressed as

$$
c_{k, m}\left(t-i T_{b}\right)=\sum_{n=0}^{N_{c}-1} c_{k, m}^{(n)}(i) \psi\left(t-i T_{b}-n T_{c}\right)
$$

where $c_{k, m}^{(n)}(i)$ is the $n$th chip of user $k$ 's signature sequence applied at the $m$ th subcarrier during the $i$ th symbol interval, which takes on values from $\left\{1 / \sqrt{N_{c}},-1 / \sqrt{N_{c}}\right\}$; equally likely, the time span of the chip-shaping waveform $\psi(t)$, which is assumed to be rectangular in this paper for simplicity [1], [10], [13], is equal to one chip interval $T_{c}=T_{b} / N_{c}$, and $N_{c}$ is the length of the signature sequence at each subcarrier. The impact of using other chip-shaping waveforms on the performance of MC-DS-CDMA systems can be found in [14]. Note that, when $N_{c}=1$, the MC-DS-CDMA with $M$ distinct signature sequences simultaneously employed over $M$ different subcarriers degenerates to an MC-CDMA system, where only one data-modulated chip is transmitted on each subcarrier for every symbol interval with $T_{c}=T_{b}$ and the length of the signature sequence is equal to the number of subcarriers [1].

The relation between MC-CDMA, MC-DS-CDMA, and single-carrier DS-CDMA can be demonstrated by defining a composite spreading ratio $N$ as the product of the number $M$ of subcarriers and the number of chips $N_{c}$ per symbol used at each subcarrier, i.e., $N=M N_{c}$ [10]. The single-carrier DS-CDMA and MC-CDMA correspond to the two extreme cases of $M=1$ and $N_{c}=1$, respectively, while MC-DS-CDMA lies in between. The composite spreading ratio can be perceived as an effective spreading gain. When conducting the performance evaluation, a common ratio $N$ should be assigned to different systems for fair comparison.

In this study, our major focus lies on performance analysis for the uplink asynchronous MC-DS-CDMA system. The analytic results obtained are also applicable to MC-CDMA with slight modifications by setting the time-domain spreading gain $N_{c}$ equal to one. Also note that, as implied in (2), we have assumed that signature sequences employed by a particular user over different subcarriers are time-synchronized, i.e., they have a common switching time.

\section{B. Channel Model}

Let us consider a multicarrier CDMA system with bandwidth $W$ experiencing a wide-sense stationary uncorrelated scattering (WSSUS) frequency-selective fading channel with impulse response $h_{k}(\tau ; t)$ for each user $k$. The channel frequency response is uniformly subdivided into $M$ subchannels, each with bandwidth $W / M$. If the number $M$ of subcarriers is sufficiently large such that the subchannel bandwidth is less than the channel coherence bandwidth $(\Delta f)_{c}$, the subdivision 
of the system bandwidth results in a roughly constant channel frequency response in each subcarrier. Hence, each subchannel exhibits a frequency-nonselective effect. From another viewpoint, the intersymbol interference (ISI) due to channel dispersion can be neglected [15, p. 814], since we have chip duration $T_{c} \approx M / W>T_{m}$ resulting in only one resolvable path and, therefore, $T_{b}=N_{c} T_{c} \gg T_{m}$ for reasonable $N_{c}$, where $T_{m} \approx(\Delta f)_{c}^{-1}$ is the delay spread of the channel. Then, the received signal of a multicarrier CDMA system contributed by the $k$ th user without the effect of noise can be represented as [2], [10], [16], [17]

$$
\begin{aligned}
r_{k}(t)=\sqrt{\frac{2 P_{k}}{M}} \sum_{m=1}^{M} \sum_{i=-\infty}^{\infty} H_{k}\left(f_{m} ; i T_{b}\right) \\
\times b_{k}(i) c_{k, m}\left(t-i T_{b}\right) e^{j 2 \pi f_{m} t}
\end{aligned}
$$

where $H_{k}(f ; t)$ denotes the Fourier transform of $h_{k}(\tau ; t)$. In the above, we assume that channel time selectivity is not severe so that $H_{k}\left(f_{m} ; i T_{b}\right)$ is unchanged over a time period of $T_{b}$.

To ensure frequency nonselectivity for each subchannel in multicarrier CDMA systems, it is often required to choose a sufficiently large number of subcarriers. However, this also results in the reduction of frequency separation $\Delta f=W / M$ between adjacent subcarriers. When the subchannel bandwidth is less than the channel coherence bandwidth $(\triangle f)_{c}$, i.e., $\triangle f<$ $(\triangle f)_{c}$, the assumption of uncorrelated fading characteristics between subchannels has to be relaxed. The statistical relation between the $m$ th and $n$th subchannel fading coefficients experienced by user $k$ can be described by the frequency correlation function defined by

$$
\Phi_{k}\left(f_{m}, f_{n}\right)=\Phi_{k}\left(f_{m}-f_{n}\right)=E\left[H_{k}\left(f_{m} ; t\right) H_{k}^{*}\left(f_{n} ; t\right)\right]
$$

where the first equality holds because of the wide-sense stationary property and the superscript * denotes the complex conjugate. Then, the statistical property of the Rayleigh fading processes among all $M$ subcarriers for the $k$ th user can be completely described by the following covariance matrix:

$$
\mathbf{R}=E\left[\mathbf{H}_{k}(t) \mathbf{H}_{k}^{H}(t)\right]
$$

where we assume identical channel statistical characteristics for all users and $\mathbf{H}_{k}(t)=\left[H_{k}\left(f_{1} ; t\right), \cdots, H_{k}\left(f_{M} ; t\right)\right]^{T}$. Since the fading process $H_{k}(f ; t)$ for user $k$ is the Fourier transform of $h_{k}(\tau ; t)$, the above frequency correlation function $\Phi_{k}(f)$ is the Fourier transform of the multipath intensity profile (MIP) [15]. In this study, the channel is assumed to have an exponential MIP with normalized unit path energy [18]. Thus, we have

$$
E\left[\left|h_{k}(\tau ; t)\right|^{2}\right]=\frac{1}{T_{d}} e^{-\frac{\tau}{T_{d}}} \mathrm{u}(\tau)
$$

where $T_{d}$ is the rms delay spread and $\mathrm{u}(\tau)$ is the unit step function. Then, the frequency correlation function becomes

$$
\Phi_{k}\left(f_{m}, f_{n}\right)=\frac{1}{1+j 2 \pi(m-n) \frac{W T_{d}}{M}} .
$$

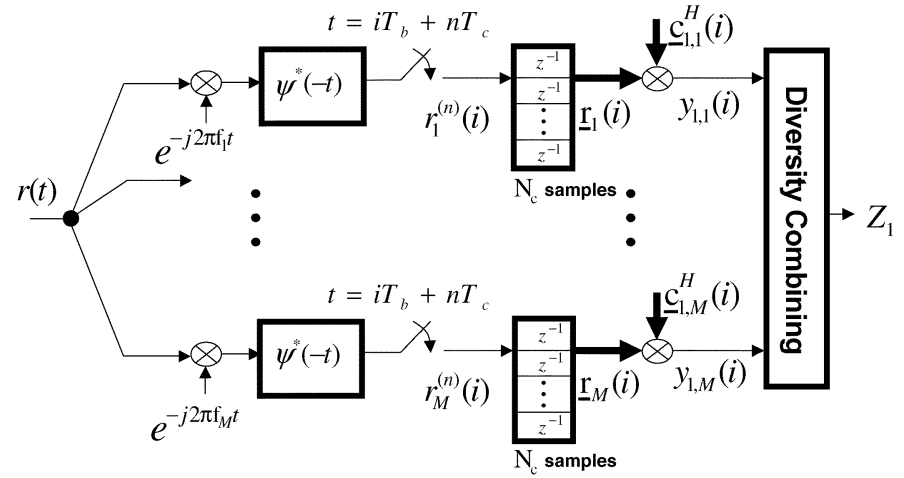

Fig. 1. Chip-matched filter and the diversity combining receiver model for the first user.

\section{Received Signal Model}

The received signal of an asynchronous multicarrier CDMA system in the presence of CFO with $K$ simultaneous users is given by

$$
r(t)=\sum_{k=1}^{K} r_{k}\left(t-\tau_{k}\right) e^{j \Omega_{k}(t)}+n(t)
$$

where $\tau_{k} \in\left[0, T_{b}\right)$ denotes the transmission delay for user $k$, $n(t)$ is the complex additive white Gaussian noise (AWGN) with $E\left[n(t) n^{*}(s)\right]=2 N_{0} \delta(t-s), N_{0}$ is the single-sided power spectral density, and $\Omega_{k}(t)=2 \pi \Delta f_{k} t+\Delta \theta_{k}$ represents the residual carrier synchronization imperfection with the frequency offset $\triangle f_{k}$ and phase shift $\triangle \theta_{k}$ for user $k$. Without loss of generality, the first user is chosen to be the user of interest. We also assume that the receiver has a perfect timing synchronization for this target user, i.e., $\tau_{1}=0$. The propagation delay $\tau_{k}$ for each interfering user can be modeled as $\tau_{k}=\left(u_{k}+v_{k}\right) \cdot T_{c}$, where $u_{k} \in\left\{0,1, \cdots, N_{c}-1\right\}$ and $v_{k} \in[0,1)$ are uniformly distributed random variables. (For MC-CDMA, $u_{k}=0$ with $\tau_{k} \in\left[0, T_{b}\right)$.) Following the receiver structure developed in [2], which is shown in Fig. 1, the received signal is first fed in parallel to $M$ downconverters, correlated with the chip-shaping waveform $\psi(t)$, and then sampled at the chip rate.

At the $m$ th subcarrier branch, the received signal vector during the $i$ th observation interval after collecting $N_{c}$ chips can be represented as

$$
\begin{aligned}
\mathbf{r}_{m}(i)= & {\left[r_{m}^{(0)}(i), r_{m}^{(1)}(i), \cdots, r_{m}^{\left(N_{c}-1\right)}(i)\right]^{T} } \\
= & \sum_{k=1}^{K} A_{k} \mathbf{F}_{k}(i) \\
& \times\left(b_{k}(i-1) \sum_{r=1}^{M} \alpha_{k, r}(i-1) \mathbf{c}_{k, m, r}^{L}(i)\right. \\
& \left.\quad+b_{k}(i) \sum_{r=1}^{M} \alpha_{k, r}(i) \mathbf{c}_{k, m, r}^{R}(i)\right)+\mathbf{n}_{m}(i)
\end{aligned}
$$


where

$$
r_{m}^{(n)}(i)=\int_{i T_{b}+n T_{c}}^{i T_{b}+(n+1) T_{c}} r(t) e^{-j 2 \pi f_{m} t} \psi^{*}\left(t-i T_{b}-n T_{c}\right) d t
$$

is the $n$th chip-matched filter output, $A_{k}=\sqrt{2 P_{k} / M} T_{c}$ accounts for the amplitude of the $k$ th user,

$$
\mathbf{F}_{k}(i)=\kappa_{k}(i) \cdot \operatorname{diag}\left\{1, e^{j 2 \pi \varepsilon_{k}}, \cdots, e^{j 2 \pi(N c-1) \varepsilon_{k}}\right\}
$$

where $\kappa_{k}(i)=e^{j\left(2 \pi i N_{c} \varepsilon_{k}+\Delta \theta_{k}\right)}$ is the scale factor depending on the timing index $i$ and $\varepsilon_{k}=\Delta f_{k} T_{c}$ denotes the normalized frequency offset with respect to the subcarrier spacing due to the $k$ th user, $\alpha_{k, r}(i)=H_{k, r}(i) e^{-j 2 \pi f_{r} \tau_{k}}$, and $\mathbf{n}_{m}(i)$ is a vector of independent complex Gaussian noise samples, each with zero mean and variance $\sigma^{2}=2 N_{0} T_{c}$.

In this study, we focus on the CFO effect only and assume $\Delta \theta_{k}=0$ for simplicity. From (6), we see that the received signal constellation is rotated and varied every symbol interval due to the presence of CFO. Specifically, the left $\mathbf{c}_{k, m, r}^{L}(i)$ and the right $\mathbf{c}_{k, m, r}^{R}(i)$ spreading vectors correspond to the effective signature sequences of the $k$ th user observed at the $m$ th subcarrier with the signal leakages from the $r$ th subcarrier. Mathematically, we have

$$
\mathbf{c}_{k, m, r}^{L}(i)=\mathbf{S}_{k, m}^{L}(i) \mathbf{t}_{k, m, r}
$$

and

$$
\mathbf{c}_{k, m, r}^{R}(i)=\mathbf{S}_{k, m}^{R}(i) \mathbf{t}_{k, m, r}
$$

where

$$
\begin{aligned}
\mathbf{S}_{k, m}^{L}(i) & =\left[\begin{array}{l}
c_{k, m}^{\left(N c-u_{k}-1\right)}(i-1), \cdot \cdot, c_{k, m}^{\left(N_{c}-1\right)}(i-1), 0, \cdot, 0 \\
c_{k, m}^{\left(N c-u_{k}\right)}(i-1), \cdot \cdot, c_{k, m}^{\left(N_{c}-1\right)}(i-1), 0,0, \cdot \cdot, 0
\end{array}\right]^{T} \\
\mathbf{S}_{k, m}^{R}(i) & =\left[\begin{array}{c}
0, \cdots, 0,0, c_{k, m}^{(0)}(i), \cdots, c_{k, m}^{\left(N_{c}-u_{k}-2\right)}(i) \\
0, \cdots, 0, c_{k, m}^{(0)}(i), \cdots, c_{k, m}^{\left(N_{c}-u_{k}-1\right)}(i)
\end{array}\right]^{T} \\
\mathbf{t}_{k, m, r} & =\left[\begin{array}{ll}
\delta_{k, m, r} & \beta_{k, m, r}
\end{array}\right]^{T}
\end{aligned}
$$

with

$$
\begin{aligned}
\delta_{k, m, r} \triangleq & \left(F_{k}^{\left(u_{k}\right)}(i)\right)^{-1} \\
& \cdot \frac{1}{T_{c}} \int_{i T_{b}+u_{k} T_{c}}^{i T_{b}+u_{k} T_{c}+v_{k} T_{c}} e^{-j 2 \pi\left(f_{m}-f_{r}-\Delta f_{k}\right) t} d t \\
= & \frac{1}{j 2 \pi\left(m-r-\varepsilon_{k}\right)}\left[1-e^{-j 2 \pi\left(m-r-\varepsilon_{k}\right) v_{k}}\right]
\end{aligned}
$$

and

$$
\begin{aligned}
\beta_{k, m, r} \triangleq & \left(F_{k}^{\left(u_{k}\right)}(i)\right)^{-1} \\
& \cdot \frac{1}{T_{c}} \int_{i T_{b}+u_{k} T_{c}+v_{k} T_{c}}^{i T_{b}+u_{k} T_{c}+T_{c}} e^{-j 2 \pi\left(f_{m}-f_{r}-\Delta f_{k}\right) t} d t \\
= & \frac{1}{j 2 \pi\left(m-r-\varepsilon_{k}\right)}\left[e^{-j 2 \pi\left(m-r-\varepsilon_{k}\right) v_{k}}-e^{j 2 \pi \varepsilon_{k}}\right]
\end{aligned}
$$

where $F_{k}^{\left(u_{k}\right)}(i)$ denotes the $u_{k}$ th entry of the diagonal matrix $\mathbf{F}_{k}(i)$. Note that we have assumed $\varepsilon_{k} \neq 0$ for all $k$, which assures nonzero denominators in finding the above $\mathbf{t}_{k, m, r}$. After some algebraic manipulations, (6) can be rewritten in a more compact form as

$$
\begin{array}{r}
\mathbf{r}_{m}(i)=\sum_{k=1}^{K} A_{k} \mathbf{F}_{k}(i) \\
\left(b_{k}(i-1) \mathbf{S}_{k}^{L}(i) \mathbf{T}_{k, m} \mathbf{h}_{k}(i-1)\right. \\
\left.+b_{k}(i) \mathbf{S}_{k}^{R}(i) \mathbf{T}_{k, m} \mathbf{h}_{k}(i)\right)+\mathbf{n}_{m}(i)
\end{array}
$$

where $\mathbf{T}_{k, m}=\operatorname{diag}\left\{\mathbf{t}_{k, m, 1}, \cdots, \mathbf{t}_{k, m, M}\right\}_{2 M \times M}$, $\mathbf{h}_{k}(i)=\left[\alpha_{k, 1}(i), \cdots, \alpha_{k, M}(i)\right]^{T}, \quad \mathbf{S}_{k}^{L}(i)=$ $\left[\mathbf{S}_{k, 1}^{L}(i), \cdots, \mathbf{S}_{k, M}^{L}(i)\right]_{N_{c} \times 2 M}, \quad$ and $\quad \mathbf{S}_{k}^{R}(i)=$ $\left[\mathbf{S}_{k, 1}^{R}(i), \cdots, \mathbf{S}_{k, M}^{R}(i)\right]_{N_{c} \times 2 M}$.

In this paper, two signature sequence assignment schemes are considered for MC-DS-CDMA systems, i.e., a common sequence or distinct sequences employed over different subcarriers of a given user. In the former, the subcarrier indexes can be dropped in, representing $\mathbf{c}_{k, m}(t), \mathbf{S}_{k, m}^{L}(i)$, and $\mathbf{S}_{k, m}^{R}(i)$.

\section{OPTIMUM COMBINING RECEIVER}

\section{A. Optimum Combining for Asynchronous MC-DS-CDMA}

The output vector collecting the despread signal from all $M$ subcarriers at the $i$ th symbol interval is given by

$$
\begin{aligned}
\mathbf{y}(i)= & {\left[\mathbf{c}_{1,1}^{H}(i) \mathbf{r}_{1}(i), \mathbf{c}_{1,2}^{H}(i) \mathbf{r}_{2}(i), \cdots, \mathbf{c}_{1, M}^{H}(i) \mathbf{r}_{M}(i)\right]^{T} } \\
= & \underbrace{A_{1} b_{1}(i) \mathbf{p}_{1}(i)}_{\triangleq \mathbf{y}_{1}(i)} \\
& +\underbrace{\sum_{k=2}^{K} A_{k}\left(b_{k}(i-1) \mathbf{D}_{k}^{L} \mathbf{T}_{k} \mathbf{h}_{k}(i-1)+b_{k}(i) \mathbf{D}_{k}^{R} \mathbf{T}_{k} \mathbf{h}_{k}(i)\right)}_{\triangleq \mathbf{y}_{\mathbf{i}}(i)} \\
& +\mathbf{n}(i)
\end{aligned}
$$

where $\mathbf{c}_{1, m}(i), 1 \leq m \leq M$, is the desired user's signature sequence employed at the $m$ th subcarrier, $\mathbf{p}_{1}(i)=\mathbf{D}_{1}^{R} \mathbf{T}_{1} \mathbf{h}_{1}(i)$ denotes the steering vector of the desired user, $\mathbf{n}(i)$ is a complex Gaussian vector with zero mean and covariance matrix $\sigma^{2} \mathbf{I}$,

$$
\begin{aligned}
& \mathbf{D}_{k}^{L}=\operatorname{diag}\left\{\mathbf{c}_{1,1}^{H}(i) \mathbf{F}_{k}(i) \mathbf{S}_{k}^{L}(i) \cdots \mathbf{c}_{1, M}^{H}(i) \mathbf{F}_{k}(i) \mathbf{S}_{k}^{L}(i)\right\}_{M \times 2 M^{2}} \\
& \mathbf{D}_{k}^{R}=\operatorname{diag}\left\{\mathbf{c}_{1,1}^{H}(i) \mathbf{F}_{k}(i) \mathbf{S}_{k}^{R}(i) \cdots \mathbf{c}_{1, M}^{H}(i) \mathbf{F}_{k}(i) \mathbf{S}_{k}^{R}(i)\right\}_{M \times 2 M^{2}}
\end{aligned}
$$


are both block-diagonal matrices with dimension $M \times 2 M^{2}$, and $\mathbf{T}_{k}=\left[\mathbf{T}_{k, 1}^{T}, \cdots, \mathbf{T}_{k, M}^{T}\right]^{T}$. When a common signature sequence is considered for each user over all different subcarriers, it yields $\mathbf{D}_{k}^{L}=\mathbf{I}_{M} \otimes\left(\mathbf{c}_{1}^{H}(i) \mathbf{F}_{k}(i) \mathbf{S}_{k}^{L}(i)\right)$ and $\mathbf{D}_{k}^{R}=\mathbf{I}_{M} \otimes$ $\left(\mathbf{c}_{1}^{H}(i) \mathbf{F}_{k}(i) \mathbf{S}_{k}^{R}(i)\right)$, where $\otimes$ denotes the standard Kronecker product and $\mathbf{c}_{1}(i)$ is the desired user's signature sequence that is identical at all subcarriers, as in the case of [2]. In the sequel, for the sake of clarity, we omit the time index $i$ in occasions without ambiguity.

The weighting vector $\mathbf{g}=\left[g_{1}, g_{2}, \cdots, g_{M}\right]^{T}$ optimally combining the output statistics from all diversity branches is determined by maximizing the SINR of the combined output, i.e.

$$
\hat{\mathrm{g}}=\arg \max _{\mathbf{g}} \frac{E\left[\left|\mathbf{g}^{H} \mathbf{y}_{1}\right|^{2} \mid \mathbf{p}_{1}\right]}{E\left[\left|\mathbf{g}^{H}\left(\mathbf{y}_{\mathbf{i}}+\mathbf{n}\right)\right|^{2}\right]}
$$

where $E[\cdot]$ denotes the expectation operator. Then, the decision is based on the sign of the real part of the combined output

$$
\hat{b}_{1}=\operatorname{sgn}\left\{\Re\left(\hat{\mathbf{g}}^{H} \mathbf{y}\right)\right\} .
$$

With the Cauchy-Schwarz inequality, the solution to (8) is

$$
\hat{\mathrm{g}}=\boldsymbol{\Sigma}^{-1} \mathbf{p}_{1}
$$

where $\boldsymbol{\Sigma}=E\left[\left(\mathbf{y}_{\mathrm{i}}+\mathbf{n}\right)\left(\mathbf{y}_{\mathrm{i}}+\mathbf{n}\right)^{H}\right]$ is the covariance matrix of the interference-plus-noise vector. The maximal value of SINR follows:

$$
\mathrm{SINR}_{\text {max }}=A_{1}^{2} \mathbf{p}_{1}^{H} \boldsymbol{\Sigma}^{-1} \mathbf{p}_{1} .
$$

Under the scenario of uncorrelated fading and perfect synchronization, the steering vector is the channel vector $\left(\mathbf{p}_{1}=\mathbf{h}_{1}\right)$ and $\boldsymbol{\Sigma}$ is a diagonal matrix, for which the optimum combining is the MRC. Also, note that the receiver does not exploit the knowledge of the interfering users' signature sequences for a reduced complexity [19].

To conduct a theoretical analysis of the optimum combining receiver, we need an exact expression for $\Sigma$. Given the knowledge of all interferers' signature sequences, CFOs and asynchronous delays, the conditional covariance matrix of $\mathbf{y}_{\mathbf{i}}$ is given by

$$
\begin{aligned}
E & {\left[\mathbf{y}_{\mathrm{i}} \mathbf{y}_{\mathrm{i}}^{H} \mid\left\{\mathbf{S}_{k}^{L}, \mathbf{S}_{k}^{R}, u_{k}, v_{k}, \varepsilon_{k} ; 2 \leq k \leq K\right\}\right] } \\
& =\sum_{k=2}^{K} A_{k}^{2}\left(\mathbf{D}_{k}^{L} \mathbf{T}_{k} \mathbf{R}_{h} \mathbf{T}_{k}^{H} \mathbf{D}_{k}^{L^{H}}+\mathbf{D}_{k}^{R} \mathbf{T}_{k} \mathbf{R}_{h} \mathbf{T}_{k}^{H} \mathbf{D}_{k}^{R^{H}}\right)
\end{aligned}
$$

where $\mathbf{R}_{h}=E\left[\mathbf{h}_{k}(i-1) \mathbf{h}_{k}^{H}(i-1)\right]=E\left[\mathbf{h}_{k}(i) \mathbf{h}_{k}^{H}(i)\right]$ because a wide-sense stationary channel is considered. Our purpose is to average all random terms in (12) to find the unconditional covariance matrix $\Sigma$.

\section{B. Closed-Form Expression of Covariance Matrix $\boldsymbol{\Sigma}$}

When the fractional part of asynchronous delay $v_{k}$ is nonzero, matrix $\mathbf{R}_{h}$ can be further decomposed as $\mathbf{R}_{h}=\mathbf{V}_{k} \mathbf{R} \mathbf{V}_{k}^{H}$, where $\mathbf{V}_{k}=\operatorname{diag}\left\{1, e^{-j 2 \pi v_{k}}, \cdots, e^{-j 2 \pi(M-1) v_{k}}\right\}$. By doing so, we separate mutually independent random parameters $\left(u_{k}, v_{k}\right)$ 's into different matrices, where the effect of integer delays $u_{k}$ 's is considered in $\mathbf{D}_{k}^{L}$ and $\mathbf{D}_{k}^{R}$, while fractional delays $v_{k}$ 's reside in $\mathbf{T}_{k}$ and $\mathbf{V}_{k}$.

We first deal with the uniformly distributed random variable $v_{k} \in[0,1)$. Since spreading codes, CFOs, $u_{k}$, and $v_{k}$ are mutually independent random variables, we can take the expectation with respect to $v_{k}$, denoted by $E_{v_{k}}[\cdot]$, in (12) and obtain

$$
\begin{aligned}
& E\left[\mathbf{y}_{\mathrm{i}} \mathbf{y}_{\mathrm{i}}^{H} \mid\left\{\mathbf{S}_{k}^{L}, \mathbf{S}_{k}^{R}, u_{k}, \varepsilon_{k} ; k \geq 2\right\}\right] \\
&=\sum_{k=2}^{K} A_{k}^{2}\left(\mathbf{D}_{k}^{L} E_{v_{k}}\left[\mathbf{T}_{k} \mathbf{V}_{k} \mathbf{R} \mathbf{V}_{k}^{H} \mathbf{T}_{k}^{H}\right] \mathbf{D}_{k}^{L^{H}}\right. \\
&\left.+\quad \mathbf{D}_{k}^{R} E_{v_{k}}\left[\mathbf{T}_{k} \mathbf{V}_{k} \mathbf{R} \mathbf{V}_{k}^{H} \mathbf{T}_{k}^{H}\right] \mathbf{D}_{k}^{R^{H}}\right)
\end{aligned}
$$

which requires finding the expectation of the $2 M^{2} \times 2 M^{2}$ matrix $E_{v_{k}}\left[\mathbf{T}_{k} \mathbf{V}_{k} \mathbf{R} \mathbf{V}_{k}^{H} \mathbf{T}_{k}^{H}\right]$. Let $\mathbf{w}_{k, r}$ be a $2 M \times 1$ vector defined by $\mathbf{w}_{k, r} \triangleq\left[\mathbf{t}_{k, r, 1}^{T}, \mathbf{t}_{k, r, 2}^{T} e^{-j 2 \pi v_{k}}, \cdots, \mathbf{t}_{k, r, M}^{T} e^{-j 2 \pi(M-1) v_{k}}\right]^{T}$ for $1 \leq r \leq M$. Then, the product $\mathbf{T}_{k, r} \mathbf{V}_{k}$ is a $2 M \times M$ block-diagonal matrix where $\mathbf{t}_{k, r, r^{\prime}}^{T} e^{-j 2 \pi\left(r^{\prime}-1\right) v_{k}}$ is its $r^{\prime}$ th diagonal block for $1 \leq r, r^{\prime} \leq M$. Based on this block-diagonal structure, we obtain the $2 M \times 2 M$ block matrix at the $(m, n)$ th block location of $E_{v_{k}}\left[\mathbf{T}_{k} \mathbf{V}_{k} \mathbf{R} \mathbf{V}_{k}^{H} \mathbf{T}_{k}^{H}\right]$ as

$$
\begin{aligned}
E_{v_{k}}\left[\mathbf{T}_{k, m} \mathbf{V}_{k} \mathbf{R} \mathbf{V}_{k}^{H} \mathbf{T}_{k, n}^{H}\right] & =E_{v_{k}}\left[\mathbf{R} \odot \mathbf{w}_{k, m} \mathbf{w}_{k, n}^{H}\right] \\
& =\mathbf{R} \odot \mathbf{W}_{m, n}^{k}
\end{aligned}
$$

where $\odot$ is defined to be the block Hadamard product and $\mathbf{W}_{m, n}^{k}=E\left[\mathbf{w}_{k, m} \mathbf{w}_{k, n}^{H}\right]$ is a $2 M \times 2 M$ matrix with the $2 \times 2$ matrix at the $(p, q)$ th block location given by

$$
\left(\mathbf{W}_{m, n}^{k}\right)_{p, q}=E_{v_{k}}\left[\mathbf{t}_{k, m, p} \mathbf{t}_{k, n, q}^{H} e^{-j 2 \pi(p-q) v_{k}}\right] .
$$

The block Hadamard product in (14) is performed in a manner that the $(p, q)$ th $2 \times 2$ block matrix of (14) is obtained from the $(p, q)$ th $2 \times 2$ block of $\mathbf{W}_{m, n}^{k}$ multiplied by the $(p, q)$ th element of $\mathbf{R}$, i.e., $\left(\mathbf{R} \odot \mathbf{W}_{m, n}^{k}\right)_{p, q}=\mathbf{R}_{p, q} \cdot\left(\mathbf{W}_{m, n}^{k}\right)_{p, q}$. As will be shown later, only the traces of (15) are of interest in either case of considering a common signature sequence or distinct sequences over different subcarriers. For each possible $m, n, p$, and $q$, we can, by evaluating (15), calculate the traces as

$$
\begin{aligned}
& \operatorname{tr}\left\{\left(\mathbf{W}_{m, n}^{k}\right)_{p, q}\right\} \\
& = \begin{cases}\frac{1}{\pi^{2}\left(m-p-\varepsilon_{k}\right)^{2}} & \text { for } m=n, p=q \\
\times\left[1+\frac{\sin \left(2 \pi \varepsilon_{k}\right)}{2 \pi\left(m-p-\varepsilon_{k}\right)}\right], & \\
\frac{1}{2 \pi^{2}\left(m-p-\varepsilon_{k}\right)\left(n-q-\varepsilon_{k}\right)} & \\
\times\left[1+\frac{\sin \left(2 \pi \varepsilon_{k}\right)}{2 \pi\left(m-q-\varepsilon_{k}\right)}+\frac{\sin \left(2 \pi \varepsilon_{k}\right)}{2 \pi\left(n-p-\varepsilon_{k}\right)}\right], & \text { for } m=n, p \neq q \\
\frac{1}{2 \pi^{2}\left(m-p-\varepsilon_{k}\right)\left(n-q-\varepsilon_{k}\right)} & \\
\times\left[1+\frac{\sin \left(2 \pi \varepsilon_{k}\right)}{2 \pi\left(m-q-\varepsilon_{k}\right)}+\frac{\sin \left(2 \pi \varepsilon_{k}\right)}{2 \pi\left(n-p-\varepsilon_{k}\right)}\right], & \text { for } m \neq n, p=q \\
\frac{1}{2 \pi^{2}\left(m-p-\varepsilon_{k}\right)\left(n-q-\varepsilon_{k}\right)} & \\
\times\left[\frac{\sin \left(2 \pi \varepsilon_{k}\right)}{2 \pi\left(m-q-\varepsilon_{k}\right)}+\frac{\sin \left(2 \pi \varepsilon_{k}\right)}{2 \pi\left(n-p-\varepsilon_{k}\right)}\right], & \text { for } m \neq n, p \neq q .\end{cases}
\end{aligned}
$$


Next, we consider the influence of random spreading sequences contributed by matrices $\mathbf{D}_{k}^{L}$ and $\mathbf{D}_{k}^{R}$. It can be easily shown that the entries of $\mathbf{D}_{k}^{L}$ are uncorrelated with those of $\mathbf{D}_{k}^{R}$ in MC-DS-CDMA systems employing either identical or distinct random sequences over different subcarriers. Thus, by taking expectation of (13) with respect to the random sequences, we have

$$
\begin{aligned}
& E\left[\mathbf{y}_{\mathbf{i}} \mathbf{y}_{\mathbf{i}}^{H} \mid\left\{\varepsilon_{k} ; k \geq 2\right\}\right] \\
& =\sum_{k=2}^{K} A_{k}^{2} \cdot E_{\left\{\mathbf{S}_{k}^{L}, \mathbf{S}_{k}^{R}\right\}}\left[\mathbf{D}_{k} E_{v_{k}}\left[\mathbf{T}_{k} \mathbf{V}_{k} \mathbf{R} \mathbf{V}_{k}^{H} \mathbf{T}_{k}^{H}\right] \mathbf{D}_{k}^{H}\right]
\end{aligned}
$$

where $\mathbf{D}_{k} \triangleq \mathbf{D}_{k}^{L}+\mathbf{D}_{k}^{R}$ and two zero matrices, i.e., $E_{\left\{\mathbf{S}_{k}^{L}, \mathbf{S}_{k}^{R}\right\}}\left[\mathbf{D}_{k}^{L} E_{v_{k}}\left[\mathbf{T}_{k} \mathbf{V}_{k} \mathbf{R} \mathbf{V}_{k}^{H} \mathbf{T}_{k}^{H}\right] \mathbf{D}_{k}^{R^{H}}\right]$ and its Hermitian, have been added without affecting the result. Note that we have removed the condition on $u_{k}$ since it does not alter the statistical distribution of $\mathbf{D}_{k}$. Let us define a cross-correlation vector

$\mathbf{d}_{k, m}^{H} \triangleq \mathbf{c}_{1, m}^{H} \mathbf{F}_{k}\left[\mathbf{S}_{k}^{L}+\mathbf{S}_{k}^{R}\right], \quad$ for $\quad k \geq 2, \quad 1 \leq m \leq M$.

Each element of $\mathbf{d}_{k, m}$ corresponds to the cross correlation between the signature sequences of the desired user and a shifted version of the interfering user at the $m$ th subcarrier in the presence of CFO. For the MC-DS-CDMA system employing a common random signature sequence over all subcarriers for a given user [2], the covariance matrix of the cross-correlation vector is given by

$$
\begin{aligned}
E\left[\mathbf{d}_{k, m} \mathbf{d}_{k, n}^{H}\right] & =\frac{1}{N_{c}}\left(\mathbf{1}_{M} \mathbf{1}_{M}^{H}\right) \otimes \mathbf{I}_{2} \\
& =\frac{1}{N_{c}}\left(\mathbf{1}_{M} \otimes \mathbf{I}_{2}\right)\left(\mathbf{1}_{M} \otimes \mathbf{I}_{2}\right)^{H}
\end{aligned}
$$

where $\mathbf{1}_{M}$ denotes the $M \times 1$ vector with all elements equal to 1 and $\mathbf{I}_{2}$ is the $2 \times 2$ identity matrix. Then, the $(m, n)$ th element of (16) yields

$$
\begin{aligned}
& \left(E\left[\mathbf{y}_{\mathbf{i}} \mathbf{y}_{\mathbf{i}}^{H} \mid\left\{\varepsilon_{k} ; k \geq 2\right\}\right]\right)_{m, n} \\
& =\sum_{k=2}^{K} A_{k}^{2} \cdot E_{\mathbf{d}_{k}}\left[\mathbf{d}_{k, m}^{H}\left(\mathbf{R} \odot \mathbf{W}_{m, n}^{k}\right) \mathbf{d}_{k, n}\right] \\
& =\frac{1}{N_{c}} \sum_{k=2}^{K} A_{k}^{2} \sum_{p, q=1}^{M} \mathbf{R}_{p, q} \cdot \operatorname{tr}\left\{\left(\mathbf{W}_{m, n}^{k}\right)_{p, q}\right\}
\end{aligned}
$$

where (18) is used to obtain (19).

On the other hand, for the system employing distinct signature sequences at different subcarriers for any given user, e.g., the degenerated case to an MC-CDMA system [1], the cross-covariance matrix of $\mathbf{d}_{k, m}$ is $E\left[\mathbf{d}_{k, m} \mathbf{d}_{k, n}^{H}\right]=\left(1 / N_{c}\right) \mathbf{I}_{2 M} \cdot \delta_{m, n}$ for $1 \leq m, n \leq M$, which gives

$$
\begin{aligned}
\left(E\left[\mathbf{y}_{\mathbf{i}} \mathbf{y}_{\mathbf{i}}^{H} \mid\left\{\varepsilon_{k} ; k \geq 2\right\}\right]\right)_{m, n} & \\
= & \frac{1}{N_{c}} \sum_{k=2}^{K} A_{k}^{2} \sum_{p=1}^{M} \operatorname{tr}\left\{\left(\mathbf{W}_{m, n}^{k}\right)_{p, p}\right\} \cdot \delta_{m, n}
\end{aligned}
$$

where we use the fact that diagonal entries of $\mathbf{R}$ are equal to one according to (5). Comparing the results in (19) and (20), we see that the nondiagonal covariance matrix $\boldsymbol{\Sigma}$ in (19) contains signal correlation among all subcarriers while the use of distinct signature sequences on the average nulls the correlation in (20). Moreover, only the traces of $\left(\mathbf{W}_{m, n}^{k}\right)_{p, q}$ are of our interest, as stated earlier.

Finally, the remaining random parameter that needs to be averaged out is the uniformly distributed frequency offset $\varepsilon_{k}$ taking values from $\left[-\varepsilon_{\max },+\varepsilon_{\max }\right]$ [5]. It is a straightforward but tedious task to carry out $E\left[\operatorname{tr}\left\{\left(\mathbf{W}_{m, n}^{k}\right)_{p, q}\right\}\right]$ with respect to $\varepsilon_{k}$. The closed-form analytic results are provided in the Appendix.

\section{PERformance AnALysis}

\section{A. Average SINR}

The average SINR, which is useful in obtaining the BEP, is derived by averaging the instantaneous SINR in (11) over the desired user's random sequences ${ }^{1}$ residing in $\mathbf{D}_{1}^{R}$. Since $v_{1}=0$, the first entry of $\mathbf{t}_{1, m, r}$ is equal to zero. In consequence, only the even numbered rows and columns of $\mathbf{T}_{1}$ and $\mathbf{D}_{1}^{R}$, respectively, are involved with the evaluation of the average SINR. Let us define the cross-correlation vector of the desired user

$$
\mathbf{d}_{m}^{H} \triangleq \mathbf{c}_{1, m}^{H} \mathbf{F}_{1} \mathbf{S}_{1}, \quad 1 \leq m \leq M
$$

where $\mathbf{S}_{1}=\left[\mathbf{c}_{1,1}, \cdots, \mathbf{c}_{1, M}\right]$ is the matrix collected from the even numbered columns of $\mathbf{S}_{1}^{R}$. We also define $\mathbf{D}_{1} \triangleq$ $\operatorname{diag}\left\{\mathbf{d}_{1}^{H}, \cdots, \mathbf{d}_{M}^{H}\right\}, \mathbf{B}_{1, m} \triangleq \operatorname{diag}\left\{\beta_{1, m, 1}, \cdots, \beta_{1, m, M}\right\}$, and $\mathbf{B}_{1} \triangleq\left[\mathbf{B}_{1,1}^{T}, \cdots, \mathbf{B}_{1, M}^{T}\right]^{T}$, yielding another representation of the average SINR given by

$$
\operatorname{SINR}_{a}=A_{1}^{2} \mathbf{h}_{1}^{H} \widetilde{\mathbf{\Sigma}} \mathbf{h}_{1}
$$

where $\widetilde{\boldsymbol{\Sigma}}=\mathbf{B}_{1}^{H} E_{\mathbf{D}_{1}}\left[\mathbf{D}_{1}^{H} \boldsymbol{\Sigma}^{-1} \mathbf{D}_{1}\right] \mathbf{B}_{1}$. It is easily shown that the $M \times M$ block matrix at the $(m, n)$ th block location of $E_{\mathbf{D}_{1}}\left[\mathbf{D}_{1}^{H} \boldsymbol{\Sigma}^{-1} \boldsymbol{D}_{1}\right]$ is

$$
\left(E_{\mathbf{D}_{1}}\left[\mathbf{D}_{1}^{H} \boldsymbol{\Sigma}^{-1} \mathbf{D}_{1}\right]\right)_{m, n}=\left(\boldsymbol{\Sigma}^{-1}\right)_{m, n} E\left[\mathbf{d}_{m} \mathbf{d}_{n}^{H}\right] .
$$

Apparently, SINR $a$ varies with different random signature sequences assigning strategies.

First, consider the case where the system employs distinct sequences over all subcarriers for each user. The off-diagonal blocks corresponding to $m \neq n$ in (22) are all zero matrices, since $\Sigma^{-1}$ is diagonal from (20). For the diagonal blocks, i.e., $m=n$, nonzero terms in $E\left[\mathbf{d}_{m} \mathbf{d}_{n}^{H}\right]$ only appear in the diagonal entries given by

$$
E\left[\mathbf{d}_{m} \mathbf{d}_{m}^{H}\right]_{m, m}=\frac{1}{N_{c}^{2}} \frac{1-\cos \left(2 \pi \varepsilon_{1} N_{c}\right)}{1-\cos \left(2 \pi \varepsilon_{1}\right)}
$$

and

$$
E\left[\mathbf{d}_{m} \mathbf{d}_{m}^{H}\right]_{p, p}=\frac{1}{N_{c}}, \quad \text { fo } \mathrm{r} p \neq m .
$$

${ }^{1}$ We do not attempt to average $\varepsilon_{1}$ here since we are interested in the behavior of the system caused by different settings of this offset. 
By plugging the above results into

$$
\widetilde{\boldsymbol{\Sigma}}_{m, m}=\sum_{n=1}^{M} E\left[\mathbf{d}_{m} \mathbf{d}_{m}^{H}\right]_{n, n}\left|\beta_{1, n, m}\right|^{2} \cdot \Sigma_{n, n}^{-1}
$$

we obtain the average SINR via (21).

Next, when the system employs a common sequence over all subcarriers for each user, the covariance matrix of $\mathbf{d}_{m}$ becomes $E\left[\mathbf{d}_{m} \mathbf{d}_{n}^{H}\right]=\left(1 / N_{c}^{2}\right)\left(1-\cos \left(2 \pi \varepsilon_{1} N_{c}\right)\right) /(1-$ $\left.\cos \left(2 \pi \varepsilon_{1}\right)\right) \mathbf{1}_{M \times M}$ for all $1 \leq m, n \leq M$. The matrix $\boldsymbol{\Sigma}$ in (21) can be obtained as

$$
\widetilde{\Sigma}=\frac{1}{N_{c}^{2}} \frac{1-\cos \left(2 \pi \varepsilon_{1} N_{c}\right)}{1-\cos \left(2 \pi \varepsilon_{1}\right)} \mathbf{B}^{H} \Sigma^{-1} \mathbf{B}
$$

where $\mathbf{B}$ is an $M \times M$ matrix where $\beta_{1, m, n}$ is the $(m, n)$ th entry.

\section{B. Average BEP}

Consider the case of independent binary transmissions of $\left\{b_{1}(i)\right\}$ with an equal a priori probability. Based on the decision rule in (9) and the asymptotic Gaussian behavior of MAI [20], the approximate BEP conditioned on the channel vector $\mathbf{h}_{1}$ is given by

$$
P_{e \mid \mathbf{h}_{1}}=E_{\mathbf{D}_{1}}\left[Q\left(\sqrt{2 \operatorname{SINR}_{\max }}\right)\right]
$$

which is difficult to obtain in a closed-form. Following the localmean approximation approach introduced in [9, App. E] and [8], the above BEP conditioned on $\mathbf{h}_{1}$ can be approximated by

$$
P_{e \mid \mathbf{h}_{1}} \approx Q\left(\sqrt{2 \operatorname{SINR}_{a}}\right)
$$

where the expression of $\mathrm{SINR}_{a}$ is derived in the previous subsection. Substituting (21) into (23) and letting $\mathbf{x}=\sqrt{2} A_{1} \mathbf{h}_{1}$ gives rise to the approximate BEP

$$
P_{e} \approx E_{\mathbf{x}}\left[Q\left(\sqrt{\mathbf{x}^{H} \widetilde{\Sigma} \mathbf{x}}\right)\right]
$$

Let $z \triangleq \mathbf{x}^{H} \widetilde{\boldsymbol{\Sigma}} \mathbf{x}$. Then, its moment generating function (MGF) $M_{z}(s)=E\left[e^{s z}\right]$ is given by

$$
M_{z}(s)=\frac{1}{\operatorname{det}\left(\mathbf{I}-s \mathbf{R}_{\mathbf{x}} \widetilde{\boldsymbol{\Sigma}}\right)}=\prod_{i=1}^{N_{e}} \frac{1}{\left(1-s \lambda_{i}\right)^{M_{i}}}
$$

where $\mathbf{R}_{x}=E\left[\mathbf{x x}^{H}\right]$, and $\left\{\lambda_{i}, i=1, \cdots, N_{e}\right\}$ represents the set of $N_{e}$ distinct eigenvalues of matrix $\mathbf{R}_{x} \widetilde{\boldsymbol{\Sigma}}$ where each $\lambda_{i}$ has multiplicity $M_{i}$. Clearly, $\sum_{i=1}^{N_{e}} M_{i}=M$.

If all of the eigenvalues of $\mathbf{R}_{x} \widetilde{\boldsymbol{\Sigma}}$ are identical, the MGF of (25) becomes $\left.M_{z}(s)=1 /(1-s \lambda)^{M}\right)$, which corresponds to the MGF of a scaled chi-squared random variable with $2 M$ degrees of freedom. This leads to an approximate BEP of (24), which is given by [21]

$$
\begin{aligned}
P_{e} & \approx \frac{1}{2}\left[1-\frac{1}{\sqrt{1+\frac{2}{\lambda}}}\left(1+\sum_{n=1}^{M-1} \frac{1 \cdot 3 \cdot 5 \cdots(2 n-1)}{n ! 2^{n}\left(\frac{\lambda}{2}+1\right)^{n}}\right)\right] \\
& \triangleq P(\lambda, M) .
\end{aligned}
$$

In general, eigenvalues of $\mathbf{R}_{x} \widetilde{\Sigma}$ are not necessarily all identical or all distinct. By partial fractional decomposition, the MGF of (25) can be decomposed to

$$
M_{z}(s)=\prod_{i=1}^{N_{e}} \frac{1}{\left(1-s \lambda_{i}\right)^{M_{i}}}=\sum_{i=1}^{N_{e}} \sum_{m=1}^{M_{i}} \frac{\rho_{i, m}}{\left(1-s \lambda_{i}\right)^{m}}
$$

where $\rho_{i, m}=\left(\left(-\lambda_{i}^{-1}\right)^{M_{i}-m} /\left(M_{i}-m\right) !\right) f_{i}^{\left(M_{i}-m\right)}\left(-\lambda_{i}^{-1}\right)$, where $f_{i}(s)=\left(1-s \lambda_{i}\right)^{M_{i}} M_{z}(s)$ and $f_{i}^{(n)}(s)$ is its $n$th derivative. Consequently, after taking the inverse Laplace transform, the pdf of of $z$ can be represented as a sum of pdfs of scaled chi-squared random variables, giving

$$
P_{e} \approx \sum_{i=1}^{N_{e}} \sum_{m=1}^{M_{i}} \rho_{i, m} P\left(\lambda_{i}, m\right)
$$

where $P\left(\lambda_{i}, m\right)$ is defined in (26). Note that the formula in (27) is implicitly parameterized by $\varepsilon_{1}$, and its numerical results provide insights into the impact of the desired user's $\mathrm{CFO}$ on the system performance. The average BEP can be obtained by averaging (27) using the Monte Carlo integration technique.

\section{Simulation Results}

In this section, we verify the accuracy of the derived analytical result for the approximate BEP and compare the performance of the optimum combining receiver with other combining techniques via computer simulation. All numerical tests were conducted under the environment set in the analytic model, i.e., perfect equal-power control, coherent detection, equal system bandwidth $W$, and perfect channel state information. A WSSUS frequency-selective Rayleigh fading channel model with the exponential MIP and the unit energy constraint was adopted. Thus, the channel frequency response correlation between any two subchannels can be determined with $W T_{d}$ in (5) serving as a parameter. To average the effects of random transmission delays and Rayleigh fading, we updated the independently generated $\tau_{k}$ 's and $\mathbf{h}_{k}$ 's every frame with a length of 200 symbols. The Monte Carlo simulation technique was employed so that the simulated BEP had a relative precision within $\sqrt{5} \times 10^{-2}$ [22]

Fig. 2(a) compares simulated and analytic results of BEP using the formula derived in (27) for the MC-DS-CDMA system with $K=20, M=4, N_{c}=32$, and $W T_{d}=2$. Distinct signature sequences over different subcarriers are adopted in this test for every user during each symbol interval. Curves in the figure demonstrate the performance with different values of $\varepsilon_{1}$. All interferers' normalized CFO $\varepsilon_{k}$ 's, $k \geq 2$, are independent 


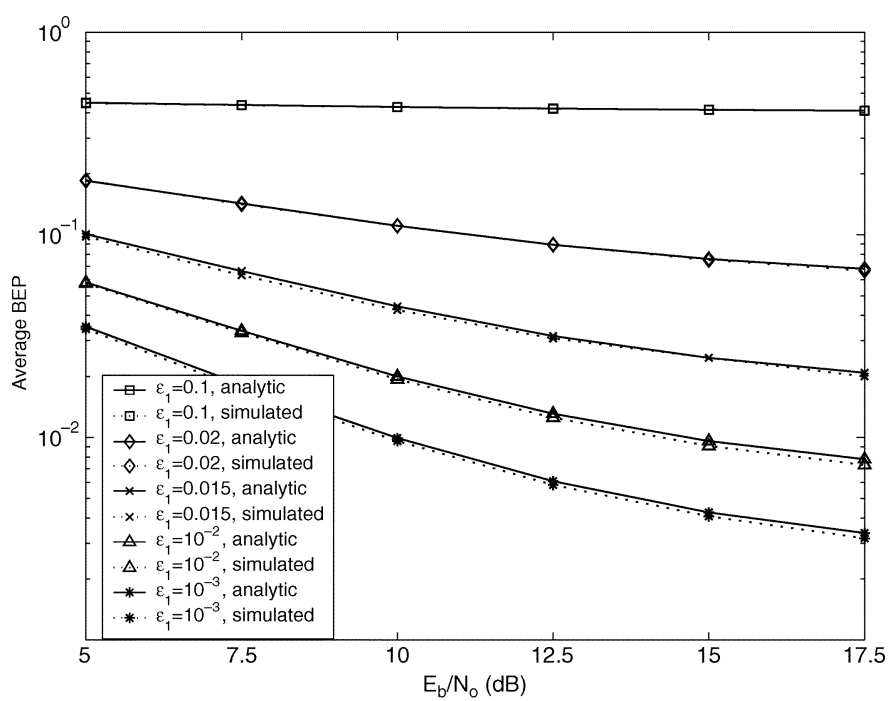

(a)

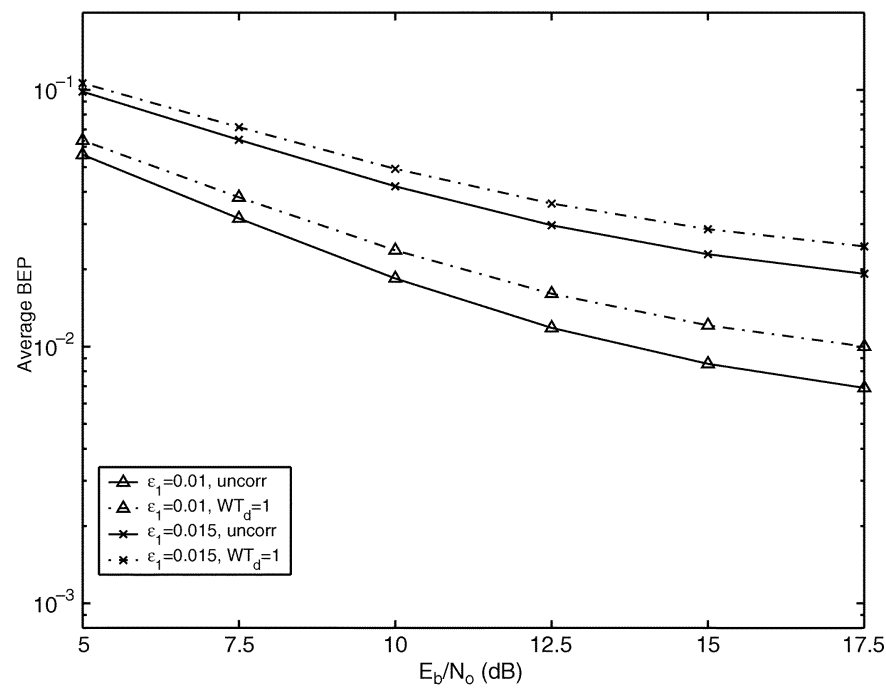

(b)

Fig. 2. (a) Effect of the desired user's CFO on the performance of an MC-DSCDMA system for $M=4, N_{c}=32$, and $K=20$, where distinct random sequences are employed to modulate different subcarriers for each user. (b) Effects of correlated fading on the performance of an MC-DS-CDMA with the same parameters as in (a).

and uniformly distributed in $\left[-\varepsilon_{\max }, \varepsilon_{\max }\right]$ with $\varepsilon_{\max }=0.5$. It can be seen that the simulated and analytic curves are close to each other. We also observe that the performance degradation due to the desired user's CFO is significant even though the optimum combining technique is employed and the SINR value is maximized. This suggests that an effective CFO estimation and compensation algorithm is critical in MC-DS-CDMA systems to ensure reliable detection. In Fig. 2(b), we illustrate the influence of correlated fading among subchannels with $M=4$, $K=20, N_{c}=32$, and $\varepsilon_{\max }=0.5$. All curves are plotted using (27). We see from this figure that the correlated fading effect impedes the effectiveness of frequency diversity, which is consistent with the intuition that a deep fade occurring in one subchannel implies weak received signals in adjacent subchannels [23].

Effects of two signature sequences assignment strategies are simulated and shown in Fig. 3(a) and (b), with $K=20$ and

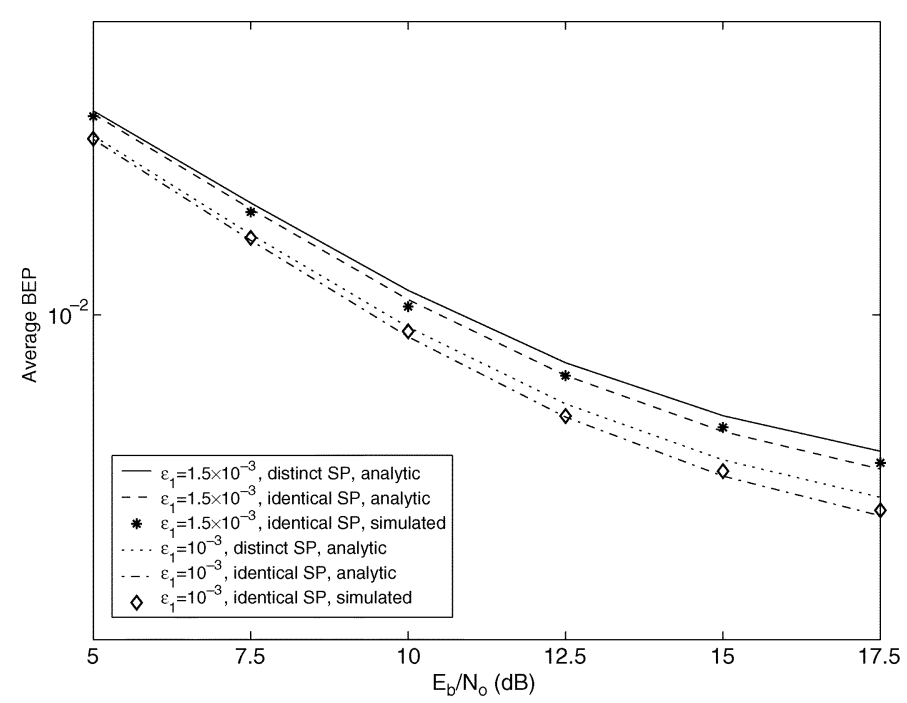

(a)

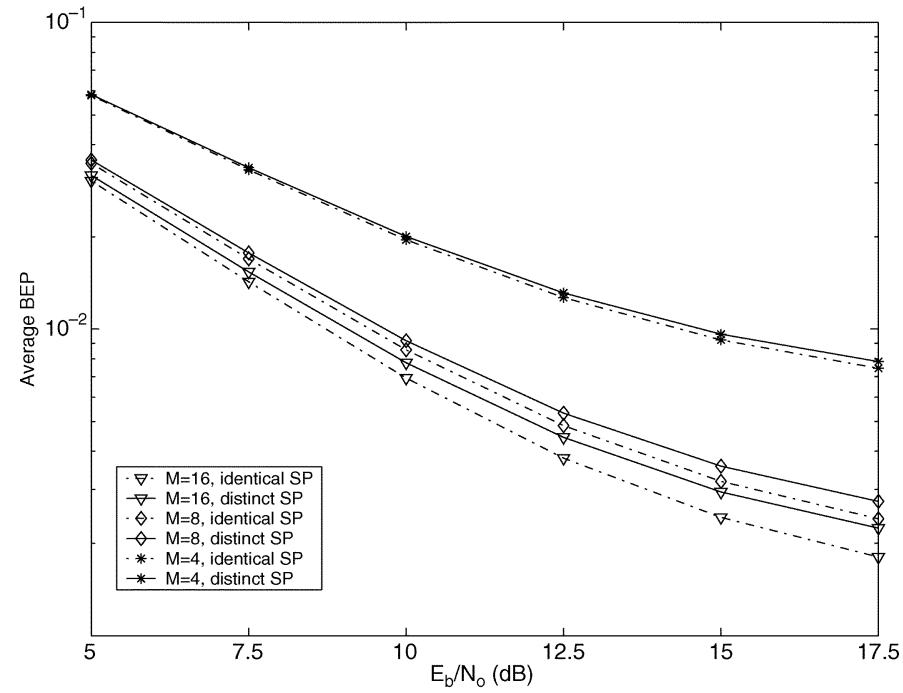

(b)

Fig. 3. (a) Simulated and analytic results of the average BEP when a common signature sequence is used at every subcarrier of a user. (b) Analytic results of the performance improvement by increasing $M$. All systems have identical composite spreading ratio $N=128, \varepsilon_{1}=0.01$, and $K=20$.

$W T_{d}=2$. We see a close match between the analytic and simulated results for the curves corresponding to the case of employing an identical spreading over all subcarriers of each user. It is also observed that the system with a common sequence over all subcarriers performs better than that with distinct ones. This can be explained as follows. When a common sequence is employed, the statistical correlation among subchannels has been exploited by the optimum combining receiver, and the impairment caused by ICI is mitigated. In contrast, the information of correlated statistics from other subchannels has been nulled out by the use of distinct uncorrelated random sequences. Moreover, as shown in Fig. 3(b), the superior performance becomes more evident as we increase the number of subcarriers, revealing that the increased ICI is further mitigated. We see from Fig. 3(b) that the MC-DS-CDMA system can achieve a potential diversity gain, when the number of subcarriers increases from $M=4$ to 8 where the composite 


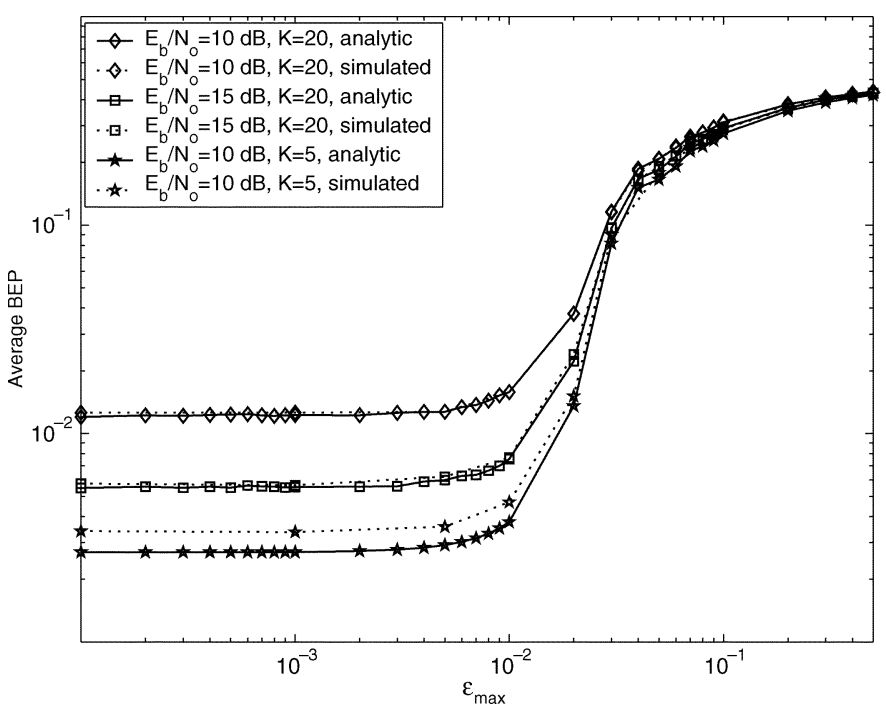

Fig. 4. BEP performance as a function of the desired user's maximal normalized CFO $\varepsilon_{\max }$ with $M=4, N_{c}=32$, and $W T_{d}=2$.

spreading ratio is a constant, even though the undesired ICI increases as well. However, increasing the number of subcarriers from $M=8$ to 16 does not improve much as compared with the previous case, since the system has gradually exhausted the diversity.

Fig. 4 shows simulated and analytic BEP performance of the MC-DS-CDMA system as a function of the desired user's $\varepsilon_{\max }$ for two differently loaded systems, with $M=4, N_{c}=32$, and $W T_{d}=2$. This experiment was conducted by varying the value of $\varepsilon_{\max }$ parameterized by different $E_{b} / N_{0}$ ratios. For each $\varepsilon_{\max }$, we generated $10^{3}$ realizations in plotting the average BEP curves based on (27) using the Monte Carlo integration. We see that simulated and analytic results are also close to each other in a heavily loaded system $K=20$. However, when the number of users reduces to $K=5$, the Gaussain assumption becomes loose in low BEP (tail) regions as the central limit theorem breaks down. It is also observed from the figure that the BEP performance degrades significantly for $\varepsilon_{\max } \geq 10^{-2}$.

Finally, Fig. 5 compares the BEP of an MC-DS-CDMA system employing the optimum combining technique with that of two other combining filters, $g=\mathbf{h}_{1}$ and $g=\Sigma^{-1} \mathbf{h}_{1}$, which correspond to the coherent combining receiver and the optimum combining receiver unaware of the presence of CFO, respectively. Similarly to the previous experiment, all curves are plotted based on (27). It is clear that the optimum combining receiver outperforms the other two receivers. Also note that the receiver making use of the MAI-plus-noise covariance matrix $\boldsymbol{\Sigma}$ performs better than that merely using the channel vector. This demonstrates that the statistical information between subchannels has been exploited to mitigate the interference.

\section{CONCLUSION}

The effects of long spreading sequences and CFO on the performance of asynchronous MC-DS-CDMA systems with correlated fading among subchannels were investigated in this paper. We obtained the closed-form solution of the optimum

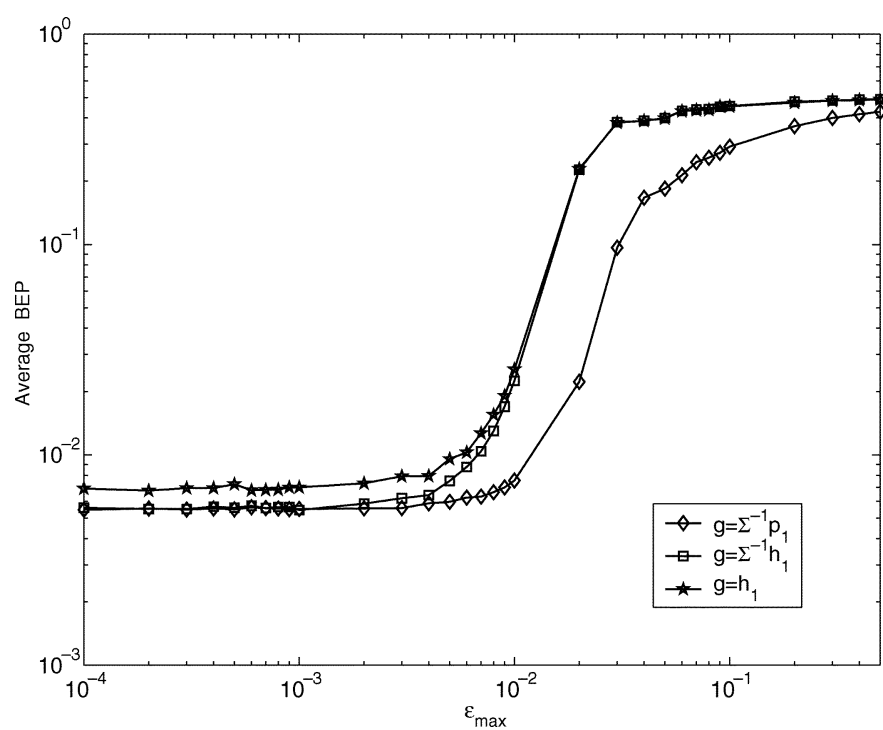

Fig. 5. BEP performance comparison using different diversity combining filters with $M=4, N_{c}=32, K=20, E_{b} / N_{0}=15 \mathrm{~dB}$, and $W T_{d}=2$.

combining filter by averaging the covariance matrix of the interference-plus-noise vector over all random parameters. An expression for approximate BEP was obtained based on Gaussian and local-mean approximation and was shown to match simulation results well in heavily loaded systems. The derived analytic results for MC-DS-CDMA with distinct signature sequences over different subcarriers for each user are applicable to MC-CDMA by appropriate modifications with $N_{c}=1$ and $u_{k}=0$. By means of numerical simulations, we demonstrated that ICI can be mitigated by using optimum combining and by assigning a common random spreading sequence over all subcarriers of a given user. The performance improvement was shown to depend on the number of subcarriers. Finally, we conclude that it is crucial to have a reliable estimate of CFO. Compensation of this offset greatly enhances system performance.

\section{APPENDIX \\ CLOSED-FORM EXPRESSION OF $E\left[\operatorname{tr}\left\{\left(\mathbf{W}_{m, n}^{k}\right)_{p, q}\right\}\right]$}

The closed-form expression of $E\left[\operatorname{tr}\left\{\left(\mathbf{W}_{m, n}^{k}\right)_{p, q}\right\}\right]$ is provided in this Appendix. For notational convenience, we define $\theta_{a, b}^{+} \triangleq \pi\left(a-b+\varepsilon_{\max }\right)$ and $\theta_{a, b}^{-} \triangleq \pi\left(a-b-\varepsilon_{\max }\right)$, where $a$ and $b$ are any possible integers. Then, the result can be carried out in the following four cases.

1) For $m=n, p=q$

$$
\begin{gathered}
E\left[\operatorname{tr}\left\{\left(\mathbf{W}_{m, n}^{k}\right)_{p, q}\right\}\right] \\
=\frac{1}{\pi}\left[\frac{1}{\theta_{m, p}^{-}}-\frac{1}{\theta_{m, p}^{+}}-\mathrm{S} i\left(2 \theta_{m, p}^{+}\right)+\mathrm{S} i\left(2 \theta_{m, p}^{-}\right)\right. \\
-\frac{\sin \left(2 \theta_{m, p}^{+}\right)}{\left(2 \theta_{m, p}^{+}\right)^{2}}-\frac{\cos \left(2 \theta_{m, p}^{+}\right)}{2 \theta_{m, p}^{+}} \\
\left.+\frac{\sin \left(2 \theta_{m, p}^{-}\right)}{\left(2 \theta_{m, p}^{-}\right)^{2}}+\frac{\cos \left(2 \theta_{m, p}^{-}\right)}{2 \theta_{m, p}^{-}}\right]
\end{gathered}
$$


2) For $m=n, p \neq q$

$$
\begin{aligned}
E\left[\operatorname{tr}\left\{\left(\mathbf{W}_{m, n}^{k}\right)_{p, q}\right\}\right] & \\
=\frac{1}{2 \pi^{2}(p-q)}[ & \ln \frac{\theta_{m, p}^{+} \cdot \theta_{m, q}^{-}}{\theta_{m, p}^{-} \cdot \theta_{m, q}^{+}}-\mathrm{C} i\left(2 \theta_{m, p}^{-}\right) \\
& +\mathrm{C} i\left(2 \theta_{m, p}^{+}\right)-\mathrm{C} i\left(2 \theta_{m, q}^{-}\right)+\mathrm{C} i\left(2 \theta_{m, q}^{+}\right) \\
& -\frac{\sin \left(2 \theta_{m, p}^{+}\right)}{2 \theta_{m, p}^{+}}+\frac{\sin \left(2 \theta_{m, p}^{-}\right)}{2 \theta_{m, p}^{-}} \\
& \left.-\frac{\sin \left(2 \theta_{m, q}^{+}\right)}{2 \theta_{m, q}^{+}}+\frac{\sin \left(2 \theta_{m, q}^{-}\right)}{2 \theta_{m, q}^{-}}\right]
\end{aligned}
$$

3) For $m \neq n, p=q$

$$
\begin{aligned}
& E\left[\operatorname{tr}\left\{\left(\mathbf{W}_{m, n}^{k}\right)_{p, q}\right\}\right] \\
& =\frac{1}{2 \pi^{2}(n-m)}\left[\ln \frac{\theta_{m, p}^{+} \cdot \theta_{n, p}^{-}}{\theta_{m, p}^{-} \cdot \theta_{n, p}^{+}}-\mathrm{C} i\left(2 \theta_{m, p}^{-}\right)\right. \\
& +\operatorname{Ci}\left(2 \theta_{m, p}^{+}\right)-\mathrm{C} i\left(2 \theta_{n, p}^{-}\right)+\mathrm{C} i\left(2 \theta_{n, p}^{+}\right) \\
& +\frac{\sin \left(2 \theta_{m, p}^{-}\right)}{2 \theta_{m, p}^{-}}-\frac{\sin \left(2 \theta_{m, p}^{+}\right)}{2 \theta_{m, p}^{+}} \\
& \\
& \left.\quad-\frac{\sin \left(2 \theta_{n, p}^{+}\right)}{2 \theta_{n, p}^{+}}+\frac{\sin \left(2 \theta_{n, p}^{-}\right)}{2 \theta_{n, p}^{-}}\right]
\end{aligned}
$$

4) For $m \neq n, p \neq q$

This case can be further divided into the following two situations.

- When $m-p=n-q$

$$
\begin{aligned}
E[\operatorname{tr} & \left.\left\{\left(\mathbf{W}_{m, n}^{k}\right)_{p, q}\right\}\right] \\
= & \frac{1}{4 \pi^{3}(p-q)^{2}} \\
& \times\left[\operatorname{Si}\left(2 \theta_{m, q}^{-}\right)-\operatorname{Si}\left(2 \theta_{m, q}^{+}\right)+2 \operatorname{Si}\left(2 \theta_{m, p}^{+}\right)\right. \\
& -2 \operatorname{Si}\left(2 \theta_{m, p}^{-}\right)-2 \mathrm{C} i\left(2 \theta_{m, p}^{-}\right)+2 \mathrm{C} i\left(2 \theta_{m, p}^{+}\right) \\
& \left.-\frac{\sin \left(2 \theta_{m, p}^{+}\right)}{\theta_{m, p}^{+}}+\frac{\sin \left(2 \theta_{m, p}^{-}\right)}{\theta_{m, p}^{-}}\right] .
\end{aligned}
$$

- Otherwise, we have

$$
\begin{aligned}
& E[\left.\operatorname{tr}\left\{\left(\mathbf{W}_{m, n}^{k}\right)_{p, q}\right\}\right] \\
&= \frac{1}{4 \pi^{3}(p-q)(n-m)} \\
& \quad \times\left[\mathrm{S} i\left(2 \theta_{m, q}^{+}\right)-\mathrm{S} i\left(2 \theta_{m, q}^{-}\right)+\mathrm{S} i\left(2 \theta_{n, p}^{+}\right)-\mathrm{S} i\left(2 \theta_{n, p}^{-}\right)\right. \\
&\left.\quad \quad \quad-\mathrm{S} i\left(2 \theta_{m, p}^{+}\right)-\mathrm{S} i\left(2 \theta_{m, p}^{-}\right)-\mathrm{S} i\left(2 \theta_{n, q}^{+}\right)+\mathrm{S} i\left(2 \theta_{n, q}^{-}\right)\right]
\end{aligned}
$$

where

$\mathrm{C} i(t)=\gamma+\ln t+\int_{0}^{t} \frac{1-\cos x}{x} d x$ and $\mathrm{S} i(t)=\int_{0}^{t} \frac{\sin x}{x} d x$

represents the standard cosine and sine integral, respectively, and $\gamma$ is the Euler constant.

\section{REFERENCES}

[1] N. Yee, J. P. Linnartz, and G. Fettweis, "Multi-carrier CDMA in indoor wireless radio networks," in Proc. IEEE PIMRC, Sep. 1993, pp. 109-113.

[2] S. Kondo and L. B. Milstein, "Performance of multicarrier CDMA systems," IEEE Trans. Commun., vol. 44, no. 2, pp. 238-246, Feb. 1996.

[3] S. Hara and R. Prasad, "Overview of multicarrier CDMA," IEEE Commun. Mag., vol. 35, pp. 126-133, Dec. 1997.

[4] L. Tomba and A. Krzymien, "Sensitivity of die MC-CDMA access scheme to carrier phase noise and frequency offset," IEEE Trans, Veh. Technol., vol. 48, no. 5, pp. 1657-1665, Sep. 1999.

[5] H. Steendam and M. Moeneclaey, "The effect of carrier frequency offsets on downlink and uplink MC-DS-CDMA," IEEE J. Sel. Areas Commun., vol. 19, no. 12, pp. 2528-2536, Dec. 2001.

[6] T. Kim, Y. Kim, J. Park, K. Ko, S. Choi, C. Kang, and D. Hong, "Performance of an MC-CDMA system with frequency offsets in correlated fading," in Proc. IEEE ICC, 2000, vol. 2, pp. 1095-1099.

[7] K. Ko, T. Kim, and D. Hong, "Performance evaluation of asynchronous MC-CDMA systems with an effect of carrier-frequency offsets," in Proc. IEEE ICC, 2003, vol. 5, pp. 3447-3451.

[8] J. P. Linnartz, "Performance analysis of synchronous MC-CDMA in mobile Rayleigh channel with both delay and doppler spreads," IEEE Trans. Veh. Technol., vol. 50, no. 6, pp. 1375-1387, Nov. 2001.

[9] T. S. Rappaport, Wireless Communications: Principles and Practice, 2nd ed. Englewood Cliffs, NJ: Prentice-Hall, 2001.

[10] S. L. Miller and B. J. Rainbolt, "MMSE detection of multicarrier CDMA," IEEE J. Sel. Areas Commun., vol. 18, no. 11, pp. 2356-2362, Nov. 2000.

[11] H. Liu and H. Yin, "Receiver design in multicarrier direct-sequence CDMA communications," IEEE Trans. Commun., vol. 49, no. 8, pp. 1479-1487, Aug. 2001.

[12] S.-M. Tseng and M. R. Bell, "Asynchronous multicarrier DS-CDMA using mutually orthogonal complementary sets of sequences," IEEE Trans. Commun., vol. 48, no. 1, pp. 53-59, Jan. 2000.

[13] S. L. Miller, M. L. Honig, and L. B. Milstein, "Performance analysis of MMSE receivers for DS-CDMA in frequency-selective fading channels," IEEE Trans. Commun., vol. 48, no. 11, pp. 1919-1929, Nov. 2000.

[14] H. H. Nguyen, "Performance of multicarrier DS-CDMA systems with time-limited chip waveforms," Can. J. Elect. Comput. Eng., vol. 29, no. 1/2, pp. 23-29, 2004.

[15] J. G. Proakis, Digital Communications, 4th ed. New York: McGrawHill, 2000.

[16] X. Gui and T. S. Ng, "Performance of asynchronous orthogonal multicarrier CDMA system in frequency selective fading channel," IEEE Trans. Commun., vol. 47, no. 7, pp. 1084-1091, Jul. 1999.

[17] T. M. Lok, T. F. Wong, and J. S. Lehnert, "Blind adaptive signal reception for MC-CDMA systems in Rayleigh fading channels," IEEE Trans. Commun., vol. 47, no. 3, pp. 464-471, Mar. 1999.

[18] W. C. Y. Lee, Mobile Communications Engineering. New York: McGraw-Hill, 1982

[19] F. T. Chien, C. H. Hwang, and C.-C. J. Kuo, "Performance of asynchronous long-code multicarrier CDMA systems in the presence of frequency offsets and correlated fading," in Proc. IEEE Globecom, 2003, vol. 2, pp. 1079-1083.

[20] D. Guo, S. Verdú, and L. K. Rasmussen, "Asymptotic normality of linear multiuser receiver outputs," IEEE Trans. Inf. Theory, vol. 48, no. 12, pp. 3080-3095, Dec. 2002.

[21] S. Verdú, Multiuser Detection. Cambridge, U.K.: Cambridge Univ. Press, 1998.

[22] M. C. Jeruchim, P. Balaban, and K. S. Shanmugan, Simulation of Communication System: Modeling, Methodology, and Tecniques, 2nd ed New York: Kluwer/Plenum, 2000.

[23] F.-T. Chien, C.-H. Hwang, C.-C. J. Kuo, and D.-C. Chang, "Performance of asynchronous long-coder multicarrier CDMA systems in the presence of correlated fading and inter-carrier interference," in Proc. IEEE ICC, 2003, vol. 3, pp. 2175-2179. 


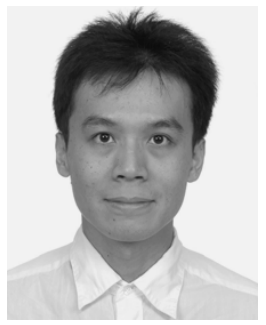

Feng-Tsun Chien received the B.S. degree from the National Tsing Hua University, Hsinchu, Taiwan, R.O.C., in 1995, the M.S. degree from the National Taiwan University, Taipei, Taiwan, R.O.C., in 1997, and the Ph.D. degree from the University of Southern California, Los Angeles, in 2004, respectively, all in electrical engineering.

He joined the Department of Electronics Engineering, National Chiao Tung University, Hsinchu, in July 2005, as an Assistant Professor. His current research interests include signal processing aspects on communications, cross-layer considerations for OFDM and OFDMA systems, multicarrier CDMA, and MIMO-OFDM systems.

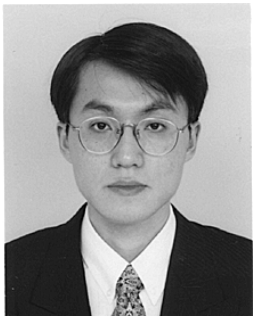

Chien-Hwa Hwang received the B.S. and M.S. degrees from the National Taiwan University, Taipei, Taiwan, R.O.C., in 1993 and 1995, respectively, and the Ph.D. degree from the University of Southern California, Los Angeles, in 2003, all in electrical engineering.

In August 2003, he joined the Institute of Communications Engineering, National Tsing Hua University, Hsnichu, Taiwan, R.O.C., as an Assistant Professor. His research interests include multiuser detection, multicarrier communications, and graph theory.

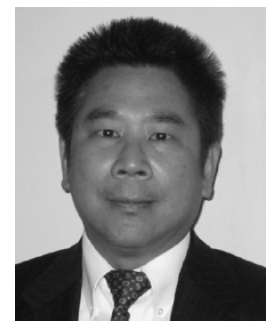

C.-C. Jay Kuo (S'83-M'86-SM'92-F'99) received the B.S. degree in electrical engineering from the National Taiwan University, Taipei, in 1980 and the M.S. and Ph.D. degrees in electrical engineering from the Massachusetts Institute of Technology, Cambridge, in 1985 and 1987, respectively.

He was Computational and Applied Mathematics (CAM) Research Assistant Professor in the Department of Mathematics, University of California, Los Angeles, from October 1987 to December 1988. Since January 1989, he has been with the Department of Electrical Engineering-Systems and the Signal and Image Processing Institute, University of Southern California, Los Angeles, where he currently has a joint appointment as Professor of Electrical Engineering and Mathematics. He has guided about 60 students to their Ph.D. degrees and supervised 15 Postdoctoral Research Fellows. He is coauthor of seven books and more than 700 technical publications in international conferences and journals. His research interests are in the areas of digital signal and image processing, audio and video coding, multimedia communication technologies and delivery protocols, and embedded system design. He is Editor-in-Chief for the Journal of Visual Communication and Image Representation and Editor for the Journal of Information Science and Engineering and the EURASIP Journal of Applied Signal Processing.

Dr. Kuo is a Fellow of SPIE and a member of ACM. He was on the Editorial Board of the IEEE Signal Processing Magazine. He served as Associate Editor for the IEEE TRANSACTIONS ON IMAGE PROCESSING from 1995 to 1998, the IEEE TRANSACTIONS ON CIRCUITS AND SYSTEMS FOR VIDEO TECHNOLOGY from 1995 to 1997, and the IEEE TRANSACTIONS ON SPEECH AND AUdiO PROCESSING from 2001 to 2003. He was the recipient of the National Science Foundation Young Investigator Award and Presidential Faculty Fellow Award in 1992 and 1993, respectively. 